\title{
RISCOS OCUPACIONAIS E PROBLEMAS DE SAÚDE PERCEBIDOS POR TRABALHADORES DE ENFERMAGEM EM UNIDADE HOSPITALAR
}

\author{
OCCUPATIONAL RISKS AND HEALTH PROBLEMS PERCEIVED BY \\ PROFESSIONAL NURSING IN HOSPITAL UNIT
}

\section{RIESGOS OCUPACIONALES Y PROBLEMAS DE SALUD PERCIBIDOS POR TRABAJADORES DE ENFERMERÍA EN UNA UNIDAD HOSPITALARIA}

\author{
Cinara Maria Feitosa Beleza* \\ Márcia Teles de Oliveira Gouveia ${ }^{* *}$ \\ Maria Lúcia do Carmo Cruz Robazzi ${ }^{* * *}$ \\ Cynthia Roberta Dias Torres ${ }^{* * * *}$ \\ Gláucia Antônia Viana de Azevedo ${ }^{* * * *}$
}

\begin{abstract}
RESUMO
Objetivou-se identificar os fatores de risco no trabalho e os problemas de saúde percebidos pelos trabalhadores de enfermagem de um instituto de doenças tropicais. Trata-se de um estudo descritivo-exploratório, com abordagem quantitativa. A amostra era composta por 99 trabalhadores de enfermagem, no ano de 2011. Para a coleta de dados aplicou-se um questionário criado por Boix e Vogel em 1997. Os dados foram analisados através do programa SPSS ${ }^{\circledR}$ versão 17.0. O estudo foi apreciado pelo Comitê de Ética em Pesquisa da Universidade Federal do Piauí. Dentre os fatores de riscos apontados, destacaram-se os biológicos, seguidos pelos riscos ergonômicos, físicos e psicossociais. Em relação aos problemas de saúde, relacionados ao trabalho, evidenciaram-se como mais frequentes: varizes, problemas osteomusculares, transtornos do sono e problemas psicossociais, como estresse edepressão.Verifica-se, então, que o ambiente de trabalho possui fatores de risco que interferem na saúde do trabalhador de enfermagem.
\end{abstract}

Palavras chave: Saúde do trabalhador, riscos ocupacionais, enfermagem.

\begin{abstract}
This study aimed to identify risk factors at work and health problems perceived by nursing staff of an institute for tropical diseases. This is a descriptive exploratory study with a quantitative approach. The sample consisted of 99 nursing staff, in the year 2011. A questionnaire created by Boix and Vogel in 1997 was applied for data collection. Data were analyzed using SPSS ${ }^{\circledR}$ version 17.0. The study was appreciated by the Ethics Committee of the Universidade Federal do Piauí. Among the risk factors pointed out, biologics aspects, followed by ergonomic, physical and psychosocial hazards, were highlighted. In relation to health problems related to work, the most frequent ones were: varicose veins, musculoskeletal problems, sleep disorders and psychosocial problems, such as stress and depression. So, then the work environment has risk factors that interfere in occupational health nursing.
\end{abstract}

Key words: Occupational health, occupational risks, nursing.

\footnotetext{
*Enfermeira. Universidade Federal do Piauí. Teresina, Brasil. Email: cinara.maria@hotmail.com

** Enfermeira. Docente da Universidade Federal do Piauí. Teresina, Brasil. Email: marcia06@gmail.com

${ }^{* * *}$ Enfermeira. Docente da Escola de Enfermagem de Ribeirão Preto da Universidade de São Paulo. Ribeirão Preto, Brasil.

Email: avrmlccr@erp.usp.br

${ }^{* * * *}$ Enfermeira. Universidade Federal do Piauí. Teresina, Brasil. Email: cynthiarobertatorres@gmail.com

${ }^{* * * * *}$ Enfermeira. Docente da Universidade Federal do Piauí. Teresina, Brasil. Email: glauciazevedo@ufpi.br
} 


\section{RESUMEN}

Este estudio tuvo como objetivo identificar los factores de riesgo en el trabajo y problemas de salud percibidos por el personal de enfermería de un instituto para enfermedades tropicales. Se trata de un estudio exploratorio descriptivo, con abordaje cuantitativo. La muestra estaba compuesta por 99 trabajadores de enfermería en 2011. Para recopilar los datos se aplicó un cuestionario creado por Boix y Vogel en 1997. Los datos fueron analizados utilizando el SPSS ${ }^{\circledR}$ versión 17.0. El estudio fue revisado por el Comité de Ética en Investigación de la Universidad Federal de Piauí. Entre los factores de riesgo mencionados se destacaron los aspectos biológicos, seguido de los riesgos ergonómicos, físicos y psicosociales. En relación con los problemas de salud relacionados con el trabajo, se mostraron como frecuentes: las venas varicosas, problemas músculo-esqueléticos, trastornos del sueño y los problemas psicosociales como el estrés y la depresión. El ambiente de trabajo tiene factores de riesgo que afectan la salud de los trabajadores de enfermería.

Palabras clave: Salud ocupacional, riesgos laborales, enfermería.

Fecha recepción: 12/10/12 Fecha aceptación: 27/08/13

\section{INTRODUÇÃO}

As doenças causadas pelo trabalho relacionam-se a natureza e as condições de execução da atividade ocupacional, aos riscos presentes nas situações de trabalho e aos modos de organização e gestão dos serviços e programas públicos (1).

Um importante fator de preservação e promoção da saúde dos trabalhadores é o ambiente em que o trabalho é realizado. Este meio deve ser saudável, tão livre quanto possível de situações de riscos e, conseqüentemente, da ocorrência de acidentes e doenças relacionadas ao trabalho, de sofrimento físico e mental, ou até mesmo da morte (2).

Os profissionais de enfermagem, inseridos na produção em saúde, estão expostos a uma diversidade de cargas produtoras de desgaste. Tratando-se especificamente do ambiente hospitalar, muito se tem discutido a respeito das condições inadequadas de trabalho vigentes em grande parte dessas instituições. Estudo aponta que os trabalhadores de enfermagem são submetidos a diferentes cargas de trabalho que geram processos de desgaste e comprometem tanto a qualidade de vida no trabalho de enfermagem, quanto a qualidade da assistência prestada (3).

Desse modo, os trabalhadores que atuam em hospitais, especialmente aqueles que se ocupam da assistência direta,em decorrência do contato com vários agentes propiciadores de riscos ocupacionais, têm grandes possibilidades de sofrer desgastes de variadas naturezas, adquirir enfermidades e sofrer acidentes de trabalho $(4,5)$.

Classicamente, os fatores de risco para a saúde e segurança dos trabalhadores, de acordo com a Organização Pan-Americana de Saúde no Brasil, podem ser classificados em cinco grupos: físicos: ruído, vibração, radiação ionizante e não-ionizante, temperaturas extremas (frio e calor), entre outros; químicos: agentes e substâncias químicas, sob a forma líquida, gasosa ou de partículas e poeiras minerais e vegetais, comuns nos processos de trabalho; biológicos: vírus, bactérias, parasitas, geralmente associados ao trabalho em hospitais; ergonômicos e psicossociais: que decorrem da organização e gestão do trabalho; de acidentes: ligados à proteção das máquinas, arranjo físico, ordem e limpeza do ambiente de trabalho, sinalização, rotulagem de produtos e outros que po- 
dem levar a acidentes de trabalho (6).

Logo, a inadequação das condições de trabalho nas instituições hospitalares, quando estas funcionam de forma improvisada ou indesejável, irá caracterizar a maior exposição dos trabalhadores aos agentes de riscos ocupacionais, incluindo os fatores de violência aos quais eles ficam expostos durante sua atividade laboral (7).

É mister destacar que o meio ambiente de trabalho relaciona-se às condições físicas, químicas, biológicas e ambientais, que determinam a atividade dos trabalhadores. Como consequência, além do adoecimento propriamente dito, as condições de trabalho e a exposição dos trabalhadores às cargas implicam em diminuição da capacidade para o trabalho, absenteísmo, elevado custo dos afastamentos, morte e qualidade da assistência prestada aos pacientes (3).

Entre os problemas de saúde mais reportados pelos trabalhadores de enfermagem são as infecções decorrentes da exposição a fluidos corpóreos e os distúrbios relacionados com o trabalho (DORT) (8). Evidenciase o câncer e o suicídio como as principais causas de morte dos enfermeiros. As neoplasias tendem a acometer sítios diversos como o cérebro, mama, fossas nasais, sistema nervoso, pele, pulmão, fígado, dentre outros, relacionadas principalmente a exposição a cargas químicas e ao trabalho noturno. Enquanto o suicídio é desencadeado pelo sofrimento no trabalho, pressão, convivência com a dor e sofrimento dos outros (9).

Nessa perspectiva, surge a necessidade de se desenvolver ações para eliminar, minimizar ou controlar os riscos de doenças e acidentes relacionados ao trabalho de enfermagem, com base nos protagonistas desse processo, por meio de uma visão holística dos sujeitos (10).

Assim, o presente estudo teve como objetivo identificar os fatores de risco no trabalho e os problemas de saúde percebidos por trabalhadores de enfermagem.

\section{MATERIAL E MÉTODO}

Tratou-se de um estudo de caráter exploratório descritivo, com abordagem quantitativa, desenvolvido em um hospital estadual de médio porte localizado no município de Teresina (PI), reconhecido como um centro de referência em medicina tropical das regióes Norte/Nordeste do Brasil. O hospital presta atendimento ambulatorial, de internação e serviços de atendimento a doenças transmissíveis.

Para realização do estudo, optou-se por uma amostra de conveniência, convidandose todos os trabalhadores que estavam presentes nos setores durante os dias de coleta de dados para participar da pesquisa. A população foi composta por 166 (cento e sessenta e seis) trabalhadores de enfermagem que atuavam nessa instituição hospitalar durante os meses de agosto a outubro de 2011. Os participantes foram selecionados segundo a livre demanda do serviço e conforme disponibilidade em participar do estudo. Dessa forma participaram da amostra final do estudo 99 (noventa e nove) sujeitos, cuyos os critérios de inclusão foram: pertencer à categoria profissional de Enfermagem e estar exercendo ativamente sua função, com tempo mínimo de seis meses de atividade profissional nesta unidade, bem como disponibilidade em participar da pesquisa.

O instrumento de coleta de dados é composto de questionários desenvolvidos a partir dos Guias de avaliação de riscos nos lugares de trabalho em indústria, criados por Boix e Vogel em 1997 e adaptado por Mauro (11). O questionário é formado respectivamente por questões para caracterização do perfil sócio-demográfico e profissional, sob a forma de uma escala tipo Likert, que visa caracterizar as condições de trabalho, incluindo o levantamento dos riscos ocupacionais na percepção dos trabalhadores e questões referentes à identificação de problemas de 
saúde dos trabalhadores e a sua relação com as condições de trabalho.

O preenchimento do instrumento de coleta de dados somente ocorreu após a leitura e assinatura do Termo de Consentimento Livre e Esclarecido pelos participantes, conforme preconização do Conselho Nacional de Saúde. O estudo obedeceu às normas da Resolução 196/96 do Ministério da Saúde e foi apreciado pelo Comitê de Ética em Pesquisa da Universidade Federal do Piauí, sob o Protocolo de Aprovação No 0228.0.045.000-11.

Os dados coletados foram digitados e tabulados no programa SPSS ${ }^{\circledR}$, versão 17.0 , uma ferramenta no tratamento de dados e análise estatística. Realizou-se análise descritiva dos dados, com emprego de estatística simples para avaliação da freqüência e intensidade dos fatores de risco no ambiente de trabalho e sintomas percebidos. Em relação a identificação dos fatores de risco, optou-se pelo uso da média dos aspectos pontuados como "sempre", "freqüentemente" e "às vezes", tendo em vista que as dimensões das condições de trabalho derivam da inter-relação entre os mesmos.

\section{RESULTADOS}

Participaram deste estudo 99 trabalhadores de enfermagem, sendo 27 enfermeiros (27,3\%), 45 técnicos (45,4\%)e 27 auxiliares de enfermagem (27,3\%).

Em relação às características sóciodemográficas da população estudada, observa-se que $93 \%$ é do sexo feminino, $64 \%$ está compreendida na faixa etária de 31 a 50 anos, $39 \%$ é solteira e $39 \%$ casada.

Neste contexto, destaca-se maior percentual de trabalhadores atuantes nos serviços de internação em enfermarias e em Unidade de Terapia Intensiva, com $46,5 \%$ e $34,3 \%$ respectivamente. Com relação ao contrato de trabalho, observa-se que a maioria é estatutário estadual (67,7\%); seguido de 30,3\% de outros tipos de contratos. Quanto ao número de vínculos empregatícios, ressalta-se que $60,6 \%$ dos trabalhadores apresentam mais de um vínculo.

No que tange ao tipo de jornada, destaca-se que $28 \%$ possuem regime de trabalho diário, 37,4\% prestam plantões diurnos e $34,3 \%$ plantões noturnos. Quanto à carga horária semanal na instituição pesquisada, $80 \%$ desses trabalhadores possui vínculo de $30 \mathrm{~h}$, seguido por $8 \%$ que cumprecarga horária de $40 \mathrm{~h}$.

Destaca-se que $31,3 \%$ possuem carga horária de $30 \mathrm{~h}$ adicionais em outras unidades. No que concerne a faixa salarial $51,4 \%$ recebe entre 1 a 2 salários mínimos ${ }^{1}$.

Os percentuais dos problemas de saúde dos trabalhadores são apresentados no gráfico 2 que demonstra a freqüência da relação entre os problemas de saúde e o trabalho.

Observou-se que as varizes, com $71,8 \%$, e as afecções osteomusculares representadas pelas lombalgias, com $52,5 \%$, foram os problemas de saúde de maior destaque. Os desgastes psíquicos como estresse/depressão foram referidos por $(30,3 \%)$, seguidos de transtornos do sono $(29,3 \%)$.

Registraram-se, ainda, problemas de articulação $(22,3 \%)$, cefaléia freqüente $(22,2 \%)$, dores musculares crônicas $(21,3 \%)$, problemas oculares $(21,2 \%)$, lesão de coluna vertebral (19,2\%) e mudança de humor/alteração de comportamento $(19,2 \%)$, como doenças provocadas ou agravadas segundo a percepção dos trabalhadores.

\section{DISCUSSÃO E CONCLUSÃO}

A amostra apresentou predominância feminina, demonstrando que a enfermagem ainda continua sendo uma profissão essencialmente desenvolvida por mulheres. O que

1 Salario mínimo vigente no Brasil em 2011: R\$545,00, correspondendo a USD 239.64. 
Gráfico 1. Distribuição proporcional dos trabalhadores segundo a percepção de existência e freqüência de fatores de risco no ambiente de trabalho do instituto, 2012.

3. Desconhecimento das saídas de emergências

6. Choque contra móveis/imóveis

9. Risco por contato elétrico

12. Exposição à umidade excessiva

15. Exposição à ruído

18. Exposiçăo à risco biológico

21. Risco de contato com substâncias químicas

24. Esforço físico que produz fadiga

27. Posturas forçadas para a realização de alguma

30. Tarefas rotineiras ou monótonas

33. Recursos insuficientes para realizar o trabalho

36. Conflito com chefia ou encarregados

39. Pouca possibilidade de promoção

42. Desconhec/Formação insufic sobre os riscos do

45. Exposição ao vírus da Hepatite

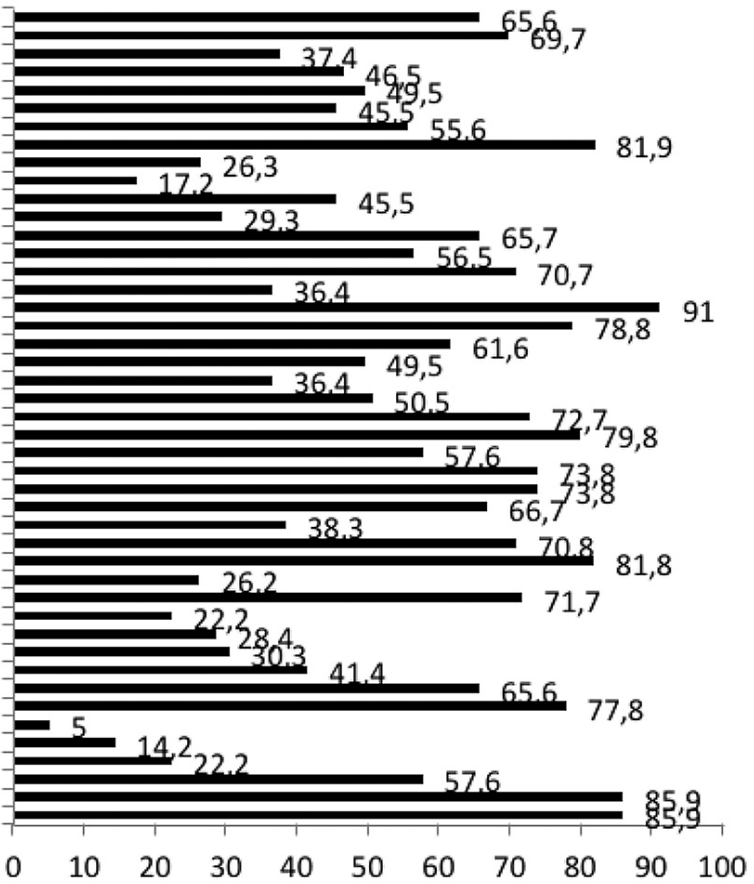

Gráfico 2. Distribuição proporcional da existência de problemas de saúde relacionados ao trabalho de acordo com os profissionais de enfermagem do instituto. Teresina - PI, 2012 (n=99).

10. Intoxicação por metais ou substâncias

12. Doenças de pele

14. Problemas difestivos

16. Problemas de articulação

18. Lesões na coluna vertebral

20. Problemas no sistema nervoso

22. Transtornos do sono

24. Alcoolismo ou uso de outras drogas

26. Afastamentos freqüentes por motivos de

28. Agressões ou condutas violentas

30. Transtornos da gravidez ou de órgão

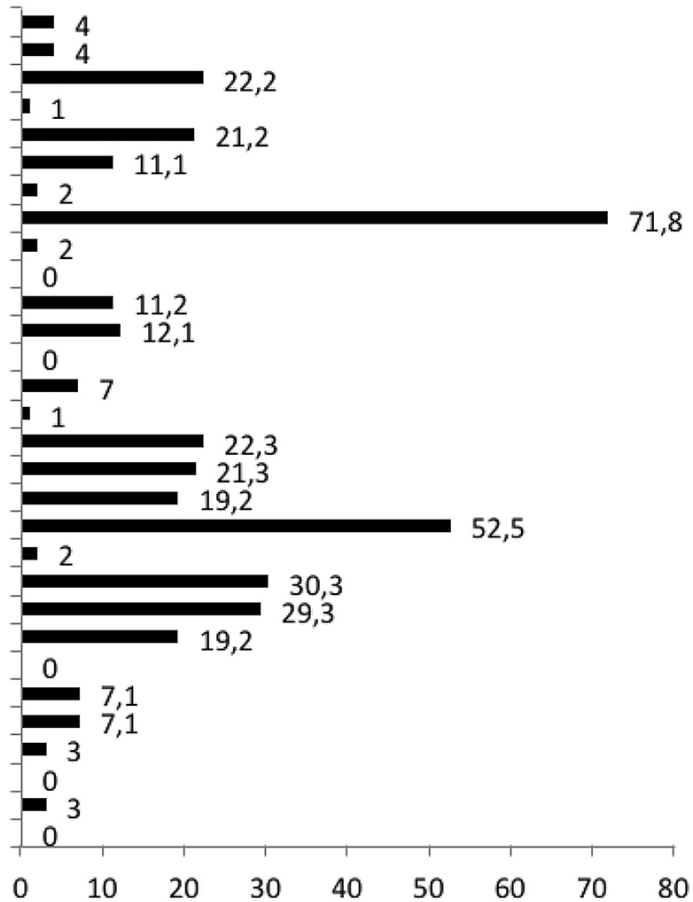


corrobora com o encontrado em hospital universitário, destacando-se que a maioria era do sexo feminino $(81,6 \%)$ (12).

Os participantes da pesquisa apresentaram uma média de idade de aproximadamente 41 anos. Este dado é relevante, uma vez que demonstra que a maioria dos trabalhadores têm mais de 40 anos, percebendo-se um aumento da faixa etária dos trabalhadores de enfermagem, que na década de 1980, estava entre 30 e 35 anos (13).

Quanto ao estado civil, há uma equivalência entre os itens solteiro e casado. É principalmente entre as mulheres que ocorrem dificuldades na interface famíliatrabalho, através do acúmulo de atividades e dificuldade em conciliar a vida familiar e profissional. Além da demanda profissional, há ainda o trabalho domiciliar (fazer compras, pagar contas, limpar a casa), entendido comumente como uma segunda jornada de trabalho (14).

Em relação a categoria profissional, destaca-se que o maior quantitativo de sujeitos refere-se à categoria de técnicos de enfermagem, o que corrobora com a realidade dos profissionais de enfermagem no Brasil, composta por um contingente maior de trabalhadores de nível médio (15).

O risco ao qual um profissional de saúde está exposto é inerente à sua atividade e à complexidade da assistência. Neste estudo evidenciou-se um maior número de profissionais atuantes nos serviços de internação em enfermarias (46,5\%), seguido pela UTI $(34,3 \%)$. Destaca-se que estas unidades apresentam ritmo diferenciado na assistência aos pacientes, quer seja pela execução de atividades repetitivas ou pelo perfil da clientela atendida. Ressalta-se ainda que o risco de exposição relaciona-se às tarefas executadas pelo trabalhador, bem como o tipo e complexidade do cuidado prestado (16).

Em relação ao contrato de trabalho, desde a promulgação da Constituição de 1988, a inserção dos trabalhadores em um órgão público dá-se através de concursos. Assim, o maior número de estatutários $(67,71 \%)$ condiz com a realidade das instituições públicas brasileiras.

No cotidiano de trabalho em hospitais, observa-se uma grande procura dos profissionais pelo trabalho noturno, que pode estar relacionada tanto aos interesses financeiros, pelo acréscimo do adicional noturno, quanto pela necessidade de conciliar atividades de ensino e vida pessoal, como por possuir outros empregos. Apesar desta procura, sabe-se que o trabalho noturno pode ser um agente causador de desconforto e problemas de saúde (17).

Com relação à carga horária semanal de trabalho da população em estudo, um total de 60 trabalhadores possui mais de um vínculo empregatício, 51,6\% desempenham atividades em outra instituição com carga horária de $30 \mathrm{~h}$ semanais, que se somadas a do hospital pesquisado, podem chegar a 60 $\mathrm{h}$ e até $70 \mathrm{~h}$ semanais, refletindo mais uma vez a sobrecarga de trabalho e identificação de cargas de trabalho que condicionam essa ocupação como penosa, perigosa e insalubre.

A dupla jornada de trabalho é ressaltada como necessária aos profissionais de enfermagem devido à situação econômica da área da saúde e aos baixos salários, insuficientes para o sustento da família; levando-os a procurar novas fontes de renda. Na realidade, eles necessitam enfrentar uma dupla atividade, o que pode interferir em alguns aspectos na qualidade de vida do trabalhador (18).

Afinal longas horas de serviço, trabalho nos turnos noturnos e finais de semana, aumentam significativamente o risco de acidentes com agulhas. Observa-se que maiores chances de acidentes com perfurocortantes em enfermeiros que trabalham muitos dias seguidos e com curto espaço de tempo entre os turnos (19).

Em pesquisa realizada num hospital especializado no atendimento de doenças transmissíveis, no interior do Estado de São Paulo, o medo da doença e sua transmissibilidade foi apontada como a maior preocupa- 
ção da equipe de enfermagem, haja vista que algumas doenças ainda não têm cura como a AIDS e a hepatite C. Como consequência deste fato, a equipe vivia diariamente sob pressão e medo dos riscos oferecidos pelo trabalho. Além disso, esses trabalhadores também se preocupavam com os familiares e as doenças que podiam levar para casa (20).

O hospital pesquisado neste estudo é especializado no atendimento a pacientes com doenças infectocontagiosas, como: meningites, leishmaniose visceral, tuberculose, tétano, hepatites, síndrome da imunodeficiência adquirida (SIDA), dentre outras; logo, a preocupação dos trabalhadores quanto algum tipo de contaminação é compreensível e real. Contudo, em estudos desenvolvidos em enfermarias de um hospital universitário, o risco de contrair infecção foi também apontado pelos profissionais de enfermagem como o mais observado nos ambientes de trabalho (12). Não sendo, por isso, um problema percebido apenas pelos trabalhadores de hospitais de doenças infectocontagiosas e parasitárias.

O risco por sobrecarga de trabalho foi outro aspecto bastante percebido pelos profissionais de enfermagem. Observa-se que o trabalho dos profissionais de enfermagem é marcado pelo tempo do relógio, pelo ritmo da demanda de usuários e pela jornada de trabalho. Além da exigência de pontualidade e regularidade, há também uma pressão para rapidez na realização das atividades, não só pela alta demanda, mas também pela necessidade de vencer a corrida em benefício da vida. O corpo do trabalhador precisa ajustar-se a rapidez pela necessidade do usuário e pelas exigências institucionais (21).

Diante dessa realidade, presente em vários países do mundo, nos Estados Unidos abordagens regulatórias, por parte de alguns estados, incluíram a proibição de horas extras obrigatórias para os enfermeiros e a definição de um número mínimo desses profissionais nas unidades de saúde. No entanto, o presente regulamento tem recebido críticas da indústria hospitalar quanto a não existência de dados suficientes que estabeleçam níveis mínimos de credibilidade pessoal de segurança (22).

Em relação aos problemas de saúde provocados ou agravados pelo trabalho, as varizes foram percebidas como as mais prevalentes. Esse distúrbio pode ser causado pelas inúmeras atividades realizadas em pé, no trabalho em enfermagem, sem pausa ou descanso. Ressalta-se que alguns fatores de risco percebidos pelos profissionais de enfermagem, como posturas forçadas $(73,8 \%)$ e desconforto pela postura adotada por muito tempo $(73,8 \%)$, podem contribuir para o desenvolvimento de varizes, além de problemas musculoesqueléticos (23).

Ao trabalhador de enfermagem, na prestação do cuidado ao cliente, muitas vezes, são exigidos empenhos que vão além de suas capacidades, tanto físicas, emocionais como mentais, e esses esforços excessivos podem desenvolver Doenças Osteomusculares Relacionadas ao Trabalho (DORT) demonstrando, assim, a vulnerabilidade dos profissionais (24).

Dentre as DORT's destacaram-se no estudo: as lombalgias, problemas de articulação, dores musculares crônicas e lesões de coluna vertebral que são, muitas vezes, associadas ao trabalho em pé, ao levantamento de pesos, trabalho com movimentos repetitivos aliados à exigência de força, posturas ergonômicas erradas, à falta de exercício e a problemas psicológicos. Evidencia-se um crescimento do adoecimento musculoesquelético do trabalhador de enfermagem, relacionado primordialmente a inadequação das condições de trabalho (25).

Os agravos decorrentes das cargas de trabalho psíquicas são expressos no corpo do trabalhador por meio da ocorrência de doenças relacionadas ao trabalho. $\mathrm{O}$ desgaste emocional, em conseqüência das tarefas, é notório, desencadeando sobrecarga emocional, com sentimentos de angústia, estresse, síndromes depressivas, entre outros agravos, 
muitas vezes associados a distúrbios físicos. Assim, as dificuldades pessoais aliadas à baixa remuneração e ao pouco prestígio social somam-se aos muitos problemas do processo de trabalho em si (26).

Neste estudo, os trabalhadores destacaram ritmo de trabalho acelerado e risco por sobrecarga de trabalho; aspectos que somados ao número de vínculos empregatícios podem ser responsáveis pelo estresse laboral. Já que, a precarização do trabalho enquanto uma realidade é geradora de estresse, desgastes, além da exposição das pessoas diretamente relacionadas à assistência, tornando insatisfatória a sua qualidade (27).

A enfermagem como profissão, para desenvolver as atividades de cuidado integral em ambiente hospitalar, necessita organizar o seu trabalho em turnos ininterruptos, acompanhando o indivíduo com problemas de saúde durante as $24 \mathrm{~h}$ do dia. Contudo, tal realidade pode acarretar problemas, afinal, os profissionais podem desenvolver alterações em seus padrões de sono habituais, nas funções fisiológicas e cognitivas que se expressam de maneira rítmica, tais como insônia, flutuações de humor, reduções no desempenho devido ao déficit de atenção, sonolência excessiva diurna e má qualidade do sono (28).

A gestão dos serviços de saúde é responsável por manter um ambiente de trabalho que contribua para aumentar a percepção de risco individual e coletivo, apoiando e orientando os profissionais em um perspectiva de maior autoproteção. Portanto, é função dos gestores proporcionar condições de trabalho adequada aos seus empregadores, expondo-os os mínimos risco (29).

Destaca-se que devido à exposição ocupacional a diversos riscos existentes no trabalho e às particularidades do processo de trabalho de enfermagem, os problemas músculo-esqueléticos têm sido apontados como um dos principais agravos de saúde apresentado por esses profissionais.

À medida que os problemas de saúde dos profissionais de enfermagem e os fatores de risco no trabalho são compreendidos torna-se possível desenvolver alternativas de intervenção que levem a mudanças em direção à apropriação pelos trabalhadores da dimensão humana do trabalho. Logo, informação, formação adequada para o trabalho e obediência às normatizações são estratégias que contribuem para a saúde ocupacional, possibilitando arealização de um trabalho de modo mais seguro e saudável.

Assim sendo, é necessário que sejam implementadas melhorias no ambiente de trabalho com a participação efetiva dos trabalhadores nas estratégias de mudanças aliadas a uma adequação ergonômica dos espaços físicos, equipamentos e mobiliários; ajuste da temperatura, do nível de ruído e da iluminação dos postos de trabalho.

Nessa perspectiva, surge a necessidade de se desenvolver ações para eliminar, minimizar ou controlar os riscos de doenças e acidentes relacionados ao trabalho de enfermagem, com base nos protagonistas desse processo, por meio de uma visão holística dos sujeitos.

\section{REFERÊNCIAS}

1. Jackson Filho JM. Considerações sobre o tema "Saúde dos Trabalhadores da Saúde" e breve apresentação. Rev. bras. saúde ocup. 2008; 33(117): 4-5.

2. Rezende MP, Robazzi MLCC, Secco IAO, Suazo SVV. Riscos físicos e sua identificação por auxiliares de enfermagem de hospital de ensino do estado de Minas Gerais, Brasil. Rev Enferm UFPE online [Internet]. 2009 [citado 22 jan 2012]; 3(3): 152-59. Disponível em: http://www. revista.ufpe.br/revistaenfermagem

3. Felli VEA. As condições de trabalho de enfermagem e adoecimento: motivos para redução da jornada de trabalho para 30 horas. Enfermagem em foco 2012; 
3(4): 178-181.

4. Costa TF, Felli VEA. Exposição dos trabalhadores de enfermagem às cargas químicas em um hospital público universitário da cidade de São Paulo. Rev Lat Am Enfermagem. 2005; 13(4): 501-8.

5. Sêcco IAO, Robazzi MLCC, Shimizu DS, Rúbio MMS. Typical occupational accidents with employees of a university hospital in the south of Brazil: epidemiology and prevention. Rev Lat Am Enfermagem. 2008; 16(5): 824-831.

6. Robazzi MLCC, Barros Júnior JC. Proposta brasileira de normatização para os trabalhadores de saúde. Cienc. enferm. 2005; XI(2): 11-15.

7. Mauro MYC, Muzi CD, Guimarães RM, Mauro CCC. Riscos ocupacionais em saúde. Rev. enferm. UERJ. 2004; 12: 33845.

8. Sarquiz LMM. O monitoramento do trabalhador de saúde após exposição de fluídos biológicos [Tese Doutorado]. [São Paulo]: Escola de Enfermagem, Universidade de São Paulo; 2007. 190 p.

9. Karino ME. As causas de morte dos enfermeiros: uma revisão sistemática [Tese Doutorado]. [São Paulo]: Escola de Enfermagem, Universidade de São Paulo; 2012. 165 p.

10. Dalri RCMB, Robazzi MLCC, Silva LA. Riscos ocupacionais e alterações de saúde entre trabalhadores de enfermagem brasileiros de unidades de urgência e emergência. Cienc. enferm. 2010; XVI(2): 6981.

11. Mauro MYC. Inovação de gestão das condições de trabalho em saúde para hospitais do Sistema Único de Saúde-SUS/ Brasil. Rio de Janeiro (RJ); 2006. Projeto apresentado ao CNPQ. Mimeografado.

12. Mauro MYC, Paz AF, Mauro CCC, Pinheiro MAS, Silva VG. Condições de trabalho da enfermagem nas enfermarias de um hospital universitário. Esc Anna Nery. 2010; 14(1): 13-18.

13. Sápia T, Felli VEA, Ciampone MHT.
Problemas de saúde de trabalhadores de enfermagem em ambulatórios pela exposição à cargas fisiológicas. Acta Paul Enferm. 2009; 22(6): 808-813.

14. Elias MA, Navarro VL. A relação entre o trabalho, a saúde e as condições de vida: negatividade e positividade no trabalho das profissionais de enfermagem de um hospital escola. Rev Lat Am Enfermagem. 2006; 14(4): 517-25.

15. Barreto IS, Krempel MC, Humerez DC. O Cofen e a Enfermagem na América Latina. Enfermagem em Foco. 2011; 2(4): 251-254.

16. Puro V, Carli GD, Petrosillo N, Ippolito G. Risk of healthcare exposure to bloodborne infection for italian workers, by job category and work area. Infect Control Hosp Epidemiol. 2001; 22(4): 20610.

17. Magalhães AMM, Martins CMS, Falk MLR, Fortes CV, Nunes VB. Perfil dos profissionais de enfermagem do turno noturno do Hospital de Clínicas de Porto Alegre. Rev HCPA. 2007; 27(2): 16-20.

18. Pafaro RC, Martino MMF. Estudo do estresse do enfermeiro com dupla jornada de trabalho em um hospital de oncologia pediátrica de Campinas. Rev Esc Enferm USP. 2004; 38(2): 152-160.

19. Trinkoff AM, Le R, Geiger-Brown J, Lipscomb J. Work schedule, needle use, and needlestick injuries among registered nurses. Infect Control Hosp Epidemiol. 2007; 28(2): 156-164.

20. Piai TH, Figueiredo RM. A co-infecção AIDS/Hepatite $\mathrm{C}$ e a equipe de enfermagem em um hospital especializado. Rev Electr Enf. [Internet]. 2009 [citado 22 jan 2012]; 11(1): 94-100. Disponível em: http://www.fen.ufg.br/revista/v11/n1/ pdf/v11n1a12.pdf

21. Costa ALRC, Marziale MHP. Relação tempo-violência no trabalho de enfermagem em Emergência e Urgência. Rev Electr Enf. 2006; 59(3): 337-43.

22. Stone PW, Clarke SP, Cimiotti J, Correa 
de Araújo, R. Nurses' working conditions: implications for infectious diseases. Emerg Infect Dis. 2004; 10(11): 19849.

23. Paz AF. Relação entre fatores de risco no ambiente hospitalar e a saúde dos trabalhadores de enfermagem. [Dissertação Mestrado]. [Rio de Janeiro]: Universidade do Estado do Rio de Janeiro, Faculdade de Enfermagem, 2009. 104 p.

24. Barboza MCN, Milbrath VM, Bielemann VM, Siqueira HCH. Doenças osteomusculares relacionadas ao trabalho (DORT) e sua associação com a enfermagem ocupacional. Rev Gaucha Enferm. 2008; 29(4): 633-8.

25. Magnago TSBS, Lisboa MTL, Griep RH. Trabalho da enfermagem e distúrbio musculoesquelético: revisão das pesquisas sobre o tema. Esc Anna Nery. 2008; 12(3): 560-5.

26. Secco IAO, Robazzi MLCC, Souza FEA, Shimizu DS. Cargas psíquicas de trabalho e desgaste dos trabalhadores de enfer- magem de hospital de ensino do Paraná, Brasil. SMAD Revista Eletrônica Saúde Mental, Álcool e Drogas [Internet]. 2010 [citado 22 nov 2013]; 6(1): 1-17. Disponível em: http://www.redalyc.org/articulo.oa?id=80313414016

27. Melo DS, Souza ACS, Tipple AFV, Neves ZCP, Pereira MS. Nurses' understanding of standard precautions at a public hospital in Goiania - GO, Brazil. Rev Lat Am Enfermagem. 2006; 14(5): 720-7.

28. Barboza JIRA, Moraes EL, Pereira EA, Reimão RNAA. Avaliação do padrão de sono dos profissionais de Enfermagem dos plantões noturnos em Unidades de Terapia Intensiva. Einstein (Sao Paulo). 2008; 6(3): 296-301.

29. Neves HCC, Souza ACS, Medeiros M, Munari DB, Ribeiro LCM, Tipple AFV. Safety of nursing staff and determinants of adherence to personal protective equipment. Rev Lat Am Enfermagem. 2011; 19(2): 354-361. 